\title{
Development of a novel phase change emulsion material for cooling systems
}

\author{
Jingjing Shao a,", Jo Darkwa a and Georgios Kokogiannakis ${ }^{\mathrm{b}}$ \\ ${ }^{a}$ Department of Architecture and Built Environment, Faculty of Engineering, University of Nottingham, \\ University Park, NG7 2RD Nottingham, UK \\ ${ }^{\mathrm{b}}$ Sustainable Buildings Research Centre, University of Wollongong, Wollongong NSW 2522, Australia \\ Correspondence information: \\ Corresponding author: Jingjing SHAO, PhD candidate
}

Add: Department of Architecture and Built Environment, University of Nottingham, University Park,

Nottingham, UK, NG7 2RD

Tel: +44 (0) 7436993953

Email: jingjing.shao@nottingham.edu.cn 


\section{Development of a novel phase change emulsion material for cooling systems}

\section{Nomenclature}

$\begin{array}{llll}\mathrm{T}_{\mathrm{m}} & \text { Melting point } & \Delta \mathrm{h}_{\mathrm{f}} & \text { Latent heat capacity } \\ \mathrm{C}_{\mathrm{p}} & \text { Specific heat capacity } & \Delta \mathrm{h}_{\mathrm{e}} & \text { Heat storage capacity } \\ \eta & \text { Viscosity } & \Delta \mathrm{h}_{\mathrm{s}} & \text { Sensible heat capacity } \\ \varphi & \text { Volume fraction } & \mathrm{T} & \text { Temperature } \\ \mathrm{X} & \text { Weight fraction } & \mathrm{V} & \text { Volume }\end{array}$

\section{Subscripts}

$\begin{array}{llll}\mathrm{e} & \text { Emulsion } & \mathrm{S} & \text { surfactant } \\ \mathrm{p} & \text { Paraffin } & \text { separate } & \text { Separated phase } \\ \mathrm{w} & \text { Water } & & \end{array}$

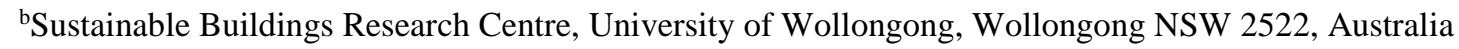

\section{Abstract}

In this paper, a novel phase change emulsion material (PCE-10) consisting of an organic segregation but the viscosity was found to be much higher than that of water.

Further improvement and experimental studies into its flow characteristics are therefore being encouraged.

Keywords: PCM emulsion; preparation method; thermo-physical properties; stability 


\section{Introduction}

19 Chilled water system is one of the most popular air conditioning systems world-wide due to its relatively low running cost [1]. However, circulating pumps are responsible for roughly $15 \%-30 \%$ of their overall energy consumption [2]. Ice slurries, as an alternative cooling media, have been widely applied in large scales. However, due to the fact that the evaporator temperature of the air conditioning system has to be lowered to produce ice slurries, the performance of air conditioner becomes worse and consequently the cold generation is more expensive [3] .

To this end, some researchers have suggested replacing water with phase change emulsion (PCME) since they possess higher heat capacities and therefore able to reduce volume flow rates and energy consumption in pumps [4]. They are multifunctional fluids which consist of Phase Change Materials (PCMs) and a carrier fluid.

However, most of the commercially available phase change emulsions do experience high levels of sub-cooling temperatures which tend to affect the performance of refrigeration equipment. For instance several studies on sub-cooling in PCME have been published including Huang et al. [5] who reported sub-cooling of $4.3 \mathrm{~K}$ and $14.7 \mathrm{~K}$ in tetradecane/water and hexadecane/water emulsions respectively. Royon and Guiffant [6] also observed a 5.6 K of sub-cooling in aliphatic hydrocarbons-water emulsion.

Another main barrier affecting the application of PCMEs is the difficulty in maintaining stability in emulsions without experiencing temperature stratification when subjected to mechanical-thermal loadings and also during a long-term storage period (roughly for 5 to 9 months when air conditioning systems are not working outside summer months). For example, tetradecane and water emulsions made by Xu et al. [7] was stable for only 40-50s.

41 Schalbart et al. [8] tested tetradecane and water emulsion and achieved a short period of

42 storage stability at room temperature. The PCME developed by Huang [9] experienced 
instability just after a short storage period of one month and 100 melting/freezing thermal cycling. Vilasau et al. [10] also tested the freeze-thaw stability of a paraffin emulsion but achieved stability for only 5 cycles.

This study is therefore intended to develop a novel phase change emulsion material that is capable of overcoming these technical barriers.

\section{Development of PCME}

\subsection{Selection of Paraffin}

According to Huang et al [9], selection of PCMEs for air conditioning application should be based on properties such as high heat capacity, low sub-cooling temperature, long term storage stability and low viscosity. Paraffin materials such as tetradecane $\mathrm{CH}_{3}-\left(\mathrm{CH}_{2}\right)_{12}-\mathrm{CH}_{3}$ $\left(\mathrm{T}_{\mathrm{m}}=5.8^{\circ} \mathrm{C}, \Delta \mathrm{h}_{\mathrm{f}}=227 \mathrm{~kJ} \mathrm{~kg}^{-1}\right)$ and pentadecane $\mathrm{CH}_{3}-\left(\mathrm{CH}_{2}\right)_{13}-\mathrm{CH}_{3}\left(\mathrm{~T}_{\mathrm{m}}=9.9^{\circ} \mathrm{C}, \Delta \mathrm{h}_{\mathrm{f}}=206 \mathrm{~kJ} \mathrm{~kg}^{-}\right.$

${ }^{1}$ ) are typical pure organic PCMs which may be combined with water to form PCMEs for cooling applications within a phase transition temperature range of $7-12^{\circ} \mathrm{C}$. However, due to the relatively high cost of pure PCM, commercially available blended paraffin such as RT6 and RT10, are widely used for practical application. It should be noted that since the RT series are mixtures of different paraffin their melting/freezing processes do not occur at specific temperatures but rather over a temperature range. selected as the core material because its melting/freezing temperature falls within the working temperature of air conditioning systems. It also has higher heat of fusion and lower subcooling temperature than RT6.

\subsubsection{Determination of Paraffin Concentration}

Thermal properties play an important role in applications of PCME systems. It is expected that the novel PCME should have a high heat storage capacity. In the temperature range from 
$\mathrm{T}_{1}$ to $\mathrm{T}_{2}$, total heat storage capacity of PCME $\Delta \mathrm{h}_{\mathrm{e}}$ is the sum of latent heat capacity of the emulsion $\Delta \mathrm{h}_{\mathrm{f}, \mathrm{e}}$, the sensible heat capacity of water $\Delta \mathrm{h}_{\mathrm{s}, \mathrm{w}}$ and that of paraffin $\Delta \mathrm{h}_{\mathrm{s}, \mathrm{p}}$ as follows [12]:

$$
\Delta h_{\mathrm{e}}=\Delta h_{\mathrm{f}, \mathrm{e}}+\Delta h_{\mathrm{s}, \mathrm{W}}+\Delta h_{\mathrm{s}, \mathrm{p}}=X_{p} \Delta h_{\mathrm{f}, \mathrm{p}}+X_{W} C_{p, W}\left(T_{2}-T_{1}\right)+X_{p} C_{p, p}\left(T_{2}-T_{1}\right)
$$

Where, $X_{w}$ and $X_{p}$ are the weight fractions of paraffin and water, $\Delta h_{f, p}$ is the heat of fusion of paraffin in the temperature range of $T_{1}$ to $T_{2} . C_{p}$, and $C_{p, p}$ are the specific heat capacity of water and the average specific heat capacity of the paraffin, respectively.

For example, in the temperature range of $7-12^{\circ} \mathrm{C}$, water has a sensible heat capacity of $20.9 \mathrm{~kJ} \mathrm{~kg}^{-1}$, while $25 \mathrm{wt} \%$ PCME has a total heat capacity around $40 \mathrm{~kJ} \mathrm{~kg}^{-1}$, which is almost 2 times as high as that of water. A PCS is expected to have a heat capacity that is a minimum of 2 times as high as that of water [13]. For PCM concentration lower than $25 \mathrm{wt} \%$, the heat of fusion of final products may not meet the above criteria. Thus, the fraction of paraffin should not be lower than $25 \mathrm{wt} \%$ for this case.

Appropriate PCME for application in air conditioning systems should also have low viscosity to ensure an acceptable level of pressure drop in pumps. Zhao [14] proposed the following equation for predicting the kinematic viscosity of a two liquid mixture.

$$
\eta=\eta_{\mathrm{w}} e^{k \varphi}
$$

Where, $\eta$ and $\eta_{w}$ are the viscosities of emulsion and water respectively, $\varphi$ is the volume fraction of oil phase and $\mathrm{k}$ is a constant which equals to 7 when $\varphi \leq 0.74$.

For PCM concentrations over $35 \mathrm{wt} \%$, the viscosity of emulsions will be 15 times that of water. A PCME is required to have a low viscosity for applications in pump systems. Thus, the paraffin weight fraction should not be more than $35 \mathrm{wt} \%$ for this case.

Based on the theoretical analysis, different samples of emulsions consisting of $25 \mathrm{wt} \%$, $30 \mathrm{wt} \%$ and $35 \mathrm{wt} \%$ concentration levels of paraffin were prepared for evaluation as summarised in Tab. 2. Analysis of the results shows that the sample containing $25 \mathrm{wt} \%$ of 
paraffin is the most appropriate type with the acceptable level of heat of fusion and highest thermal conductivity. It also has the lowest viscosity value and therefore suitable for reducing energy consumption in pumping systems.

\section{Table 2}

\subsection{Selection of Surfactant}

Since paraffin and water are immiscible, the emulsion has to be stabilised with surfactant to prevent segregation of different components. The Hydrophile-Lipophile Balance (HLB) method was therefore adopted to determine the appropriate type of surfactants needed to stabilize the RT10/water emulsion. The principle of this method is to match the specific HLB requirement (RHLB) of the oil phase against the HLB value of the surfactant in order to obtain an optimum emulsification. HLB values can range from 0 to 20 depending upon the composition of the surfactant. As the HLB value increases, the surfactant becomes more hydrophilic and less lipophilic. In a situation where two or more surfactants are mixed together, the resulting HLB may also be calculated by using the following formula $[\underline{15}, \underline{16}]$ :

$$
H L B=X_{S, 1} \times H L B S, 1+X_{S, 2} \times H L B S, 2+\ldots \ldots
$$

\subsubsection{Determination of RHLB}

For the required HLB of oil phase, a series of emulsions will be made. Each emulsion will have the same amount of oil phase, water and surfactants but at a different HLB value. Any pair of non-ionic surfactants can be used to determine the appropriate values of HLB but one of them should have a low HLB number $(<6)$ and the other a high HLB number $(>14)$. In this study, two types of surfactants Brij $52(\mathrm{HLB}=5)$ and Tween $20(\mathrm{HLB}=16.7)$ were selected to produce five different emulsions and Eq. (3) was used to obtain their corresponding HLB values. The blended samples were then kept in test tubes and observed over a period of time in order to evaluate their chemical stability levels. As summarised in Tab. 3 sample No. 3 
117 achieved the longest period of chemical stability. Based on this result, the HLB requirement

118 of the system is closest to 10.9 .

Table 3

\subsubsection{Optimised blend of surfactants}

121 Even though sample No. 3 appears to be the best candidate, it may not be the best

122 combination as their chemical structures can affect the stability of the emulsions. For instance, surfactants with longer hydrocarbon tails are more effective in stabilizing dispersions than those with shorter carbon chain lengths [17]. For these reasons different types of non-ionic surfactant and an ionic surfactant (HTAB) with mixture HLB values around 10.9 were blended and tested for stability after 48-hour storage period. This was assessed through their separation rates by dividing the sum of the volume of water separated in the test tubes plus that of paraffin $\left(V_{\text {seperate }}=V_{w}+V_{p}\right)$ by the total sample volume $\left(\mathrm{V}_{\text {total }}=\right.$ $\left.V_{w}+V_{p}+V_{e}\right)$. This is explained in Fig. 1 and expressed mathematically in Eq. (4) as:

$$
V \%=V_{\text {seperate }} / V_{\text {total }} \times 100 \%=V_{w}+V_{p} / V_{w}+V_{p}+V_{e} \times 100 \%
$$
achieved the highest level of stability without any sign of separation after the monitored period and was attributed to their combined long hydrocarbon tails of 18 and 16 carbon atoms respectively in the alkyl chain. This is further supported by previous research [17] which indicated that long hydrocarbon tail can also act as nucleation centre for activating crystallization and for reducing sub-cooling. To this end, $60 \%$ Tween60 and 40\% Brij52

137 surfactants were selected for the optimum surfactant blend. 


\subsubsection{Concentration of Surfactant}

141 Regarding the level of concentration of surfactant, Wang et al [18] suggested that 10-25\%

142 of dispersed phase of surfactant would be effective in ensuring the stability of PCME under

143 either storage or mechanical-thermal cycling condition. However, other investigations

144 revealed that, concentration level should not be lower than $5 \mathrm{wt} \%$ of total emulsion mixture

$145[\underline{19}, \underline{20]}$.

146 In order to establish the appropriate level of concentration, 25\% RT10 and water

147 emulsions with different mass fractions of the blended surfactant (5-20\% of oil phase) were

148 prepared and tested for storage stability over a period of 72 hours. As summarised in Tab. 5,

149 sample No. 4 achieved the best stability result with no sign of chemical separation whereas

150 samples No. 1-3 suffered from rapid chemical separation of various components before the

151 end of testing period. Sample No. 5 (20\% Surfactant/oil phase ratio) produced the worst

152 result with large amount of bubbles during the emulsification process and was therefore

153 considered unsuitable for testing.

Table 5

\section{$155 \quad 2.3 \quad$ Preparation of PCME}

156 Phase change emulsion was prepared by the so-called "mixing film synthesis". All the

157 constituents were initially weighted by a precision weighing scale as given in Tab. 6 . The

158 lipophilic surfactant Brij 52 was then dissolved in RT10 (Mixture A) while hydrophilic

159 surfactant Tween 60 was mixed with deionized water (Mixture B). The mixtures were heated

160 separately to about $50-55^{\circ} \mathrm{C}$ before being added together and pre-emulsified using a

161 magnetic hot plate stirrer at a speed of 500rpm and at $50^{\circ} \mathrm{C}$ for 15 minutes. Final

162 emulsification was carried out by passing the coarse emulsion through a rotor-stator machine

163 type ULTRA-TURRAX T18 at an emulsifying speed of 7200rpm for another 45 minutes. 
164 The developed sample which was named as PCE-10 could be described as a white milky Oilin-Water emulsion.

Table 6

167 3. Characterisation of developed emulsion (PCE-10)

\section{$168 \quad 3.1 \quad$ Particle Sizing}

169 Particle sizing and their distribution profiles are important parameters for specifying emulsions. Normally, dispersions with smaller particle sizes represent increased stability than those with coarse droplets. On the other hand, viscosity generally increases significantly with smaller particle sizes for a given mass or volume concentration in the dispersed phase. This is confirmed by Schramm et al. [21] which reported that the size and characteristics of particles do affect the stability, viscosity and thermal properties of emulsions. In this regard, a laser particle size analyser was used to establish the size of the PCE-10 and as a reference for the stability test. As presented in Fig. 2, the mean particle size was obtained as $3.14 \mu \mathrm{m}$.

\subsection{Thermal Conductivity}

179 The thermal conductivity of the sample was determined with a KD2 Pro Thermal Properties 180 Analyser, which has the ability to test liquid samples with accuracy of $\pm 5 \%$. After repeated

181 tests the average thermal conductivity of the PCE-10 was obtained as $0.4 \mathrm{~W} \mathrm{~m}^{-1} \mathrm{~K}^{-1}$ at a 182 temperature of $25^{\circ} \mathrm{C}$ as summarised in Tab. 7.

\section{$184 \quad 3.3 \quad$ Phase Transition Temperature and Heat Capacity}

185 The phase change temperature and the heat of fusion tests were conducted on 5mg sample 186 of the PCE-10 with a Differential Scanning Calorimetry (DSC) apparatus type EXSTAR SII 187 DSC6220 at a scanning rate of $1^{\circ} \mathrm{C} \mathrm{min}^{-1}$. 
In a DSC, the difference in heat flow to the sample and reference at the same temperature, is recorded as a function of temperature.

$$
\frac{d H}{d t}=\left(\frac{d H}{d t}\right)_{\text {sample }}-\left(\frac{d H}{d t}\right)_{\text {reference }}
$$

The use of the extrapolated peak onset temperature $T_{e}$, end temperature $T_{c}$ and the peak temperature $T_{p}$ from DSC measurements is recommended when reporting the melting and freezing peak characteristics. $\mathrm{T}_{\mathrm{e}}$ is the intersection between the tangent to the maximum rising slope of the peak and extrapolated sample baseline. Here, the phase change temperature range is defined as the temperature difference between $T_{e}$ and $T_{c}$ of a DSC curve. The integral under the DSC peak, above the base line, gives the heat of fusion for the process:

$$
\int\left(\frac{d H}{d t}\right) d t=\Delta h_{f, p}
$$

As presented in Fig. 3, an average value for the heat of fusion was obtained as $30.5 \mathrm{~kJ} \mathrm{~kg}^{-1}$ capacity was calculated as $60.5 \mathrm{~kJ} \mathrm{~kg}^{-1}$ which is 1.8 times of water i.e. $33.4 \mathrm{~kJ} \mathrm{~kg}^{-1}$ within the same temperature range.

\subsection{Sub-cooling temperature}

Sub-cooling phenomenon is one of the issues which limit the applications of PCME. It lowers the crystallization temperature of a liquid or a gas, thus latent heat is released at a lower temperature or in a wider temperature range [22]. A large sub-cooling is not acceptable because it would enlarge the operating temperature range of a PCS system and thus deteriorates the system performance and reduces energy efficiency.

210 The sub-cooling test was conducted with the T-history method which is normally used in

211 measuring the thermal properties of bulk materials [23]. As demonstrated in Fig. 4, one of the 
212 test tubes was filled with PCM and the other with a reference material, usually water, due to its

213 well-known thermo-physical properties. The tubes were then preheated above the melting

214 temperature of the PCM and then simultaneously exposed to the ambient temperature to obtain

215 the temperature history (T versus t) curves. A typical T-history curve of PCM with sub-cooling 216 is as presented in Fig. 5. The $\Delta \mathrm{T}_{\mathrm{m}}\left(=\mathrm{T}_{\mathrm{e}}-\mathrm{T}_{\mathrm{c}}\right)$ is the sub-cooling degree.

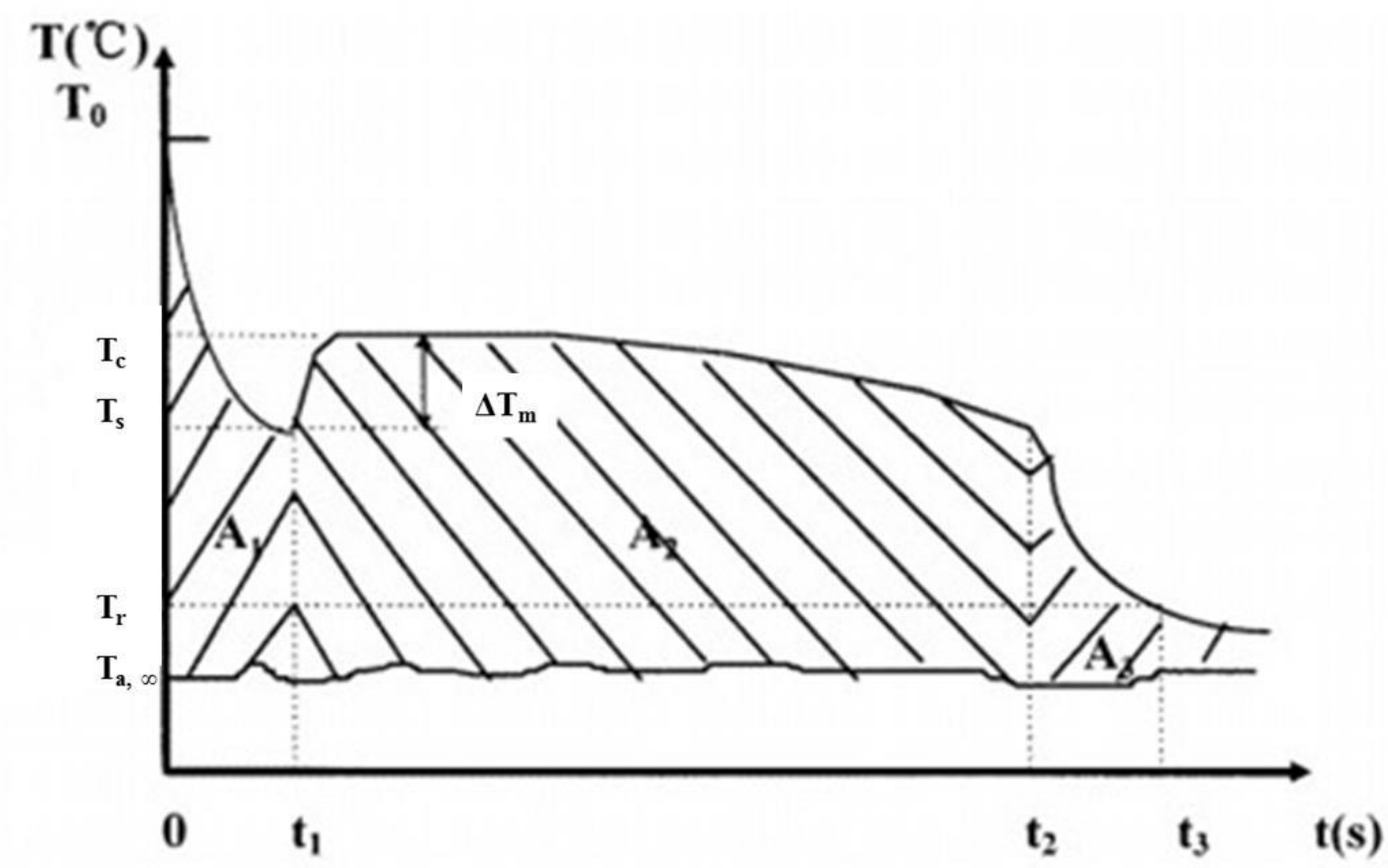
sub-cooling degree of $0.2^{\circ} \mathrm{C}$ in the developed sample.

Figure 6

\subsection{Viscosity Test}

Kinexus $\mathrm{Lab}^{+}$Rotational Rheometer was used to determine the viscosity of PCE-10. The

225 plate diameter is $50 \mathrm{~mm}$, the cone diameter is $50 \mathrm{~mm}$, and the cone angle is $1^{\circ}$. Fig. 7

226 illustrates the relationship between viscosity and shear rate for the developed PCME at $25^{\circ} \mathrm{C}$ and $10^{\circ} \mathrm{C}$. The emulsion is a pseudo-plastic fluid and shows a shear thinning behaviour. The 
viscosity of the emulsion reduced rapidly with increasing shear rate but stabilised at around $13 \mathrm{mPas}$ after $600 \mathrm{~s}^{-1}$. However, the viscosity appears quite high since it is about 13 times higher than that of water.

Figure 7

The non-Newtonian behaviour can be described using Ostwald Equation:

$$
\eta=K \times \gamma^{n-1}
$$

Where, $\eta$ is the viscosity, $\gamma$ is the shear rate. $K$ and $n$ are coefficients which can be determined with a rheometer. The coefficient $\mathrm{n}$ is the flow behaviour index of a fluid which characterizes its degree of non-Newtonian behaviour. The greater the divergence of $\mathrm{n}$ from unity in either direction, the more non-Newtonian is the fluid. For Newtonian fluids, $n=1$ and for pseudo-plastic fluids $n<1$. The coefficient $K$, flow consistency index, defines the fluid's consistency. The larger the value of $\mathrm{K}$, the thicker is the fluid.

The coefficients $\mathrm{K}$ and $\mathrm{n}$ of PCE-10 were obtained by interpreting the curves in Fig. 7 with the Ostwald Equation Eq. (5). The obtained $\mathrm{K}$ and $\mathrm{n}$ are shown in Tab. 8

Table 8

\subsection{Stability Test}

Non-cycling mode

The emulsions were stored in containers made of glass at room temperature. The test was carried out at a room temperature through visual inspection and over periodic number of days. As presented in Fig. 8, separation started to occur after 90 days of storage and by the end of 270 days there was a clear separation between the upper and lower layers. This phenomenon is called creaming and it is caused by the fact that paraffin has a lower density than water. Creaming is the movement of oil droplets under gravity or in a centrifuge to form a concentrated layer at the top of an oil-in-water emulsion. Particle size distribution of sample was tested at the end of the storage period. The result shows a value of $3.41 \mu \mathrm{m}$ (see Fig. 9) at 
the end of 270 days storage as compared with the original value of $3.14 \mu \mathrm{m}$. This indicates the presence of oil droplets coalescence.

Cycling mode

The stability of the sample was examined after being subjected to a number of thermal and mechanical loadings. The test rig as illustrated in Fig. 10, consists of a storage tank, a heat exchanger and a circulating pump. The sample was initially cooled down from $25^{\circ} \mathrm{C}$ to $5^{\circ} \mathrm{C}$ and then reheated to $15^{\circ} \mathrm{C}$. After 500 cycles, a clear layer of oil was observed (i.e. oiling-off phenomena) as shown in Fig. 11 at the surface of the sample which was a sign of separation of the base material (RT10) from the emulsion.

The droplet size distribution, viscosity and thermal properties of the samples were then analysed and compared with the data before cycling. As shown in Fig. 12, there was a slight increase in viscosity from $13 \mathrm{mPas}$ to $16 \mathrm{mPas}$ at $500 \mathrm{rpm}$. The droplet size also increased slightly from the original size of $3.14 \mu \mathrm{m}$ to $3.93 \mu \mathrm{m}$ (see Fig. 13). The latent heat of fusion was reduced from 30.5 to $29.9 \mathrm{~kJ} \mathrm{~kg}^{-1}$ as illustrated in Fig. 14. This was attributed to the fact that when the emulsion was heated above the melting point, the crystallized droplets melted and fused together thus leading to coalescence and oiling-off at the surface of the emulsion. Although some changes were observed in the properties, the emulsion remained in dispersion and maintained the heat capacity after the test. 


\section{Conclusion}

In this paper, a novel paraffin-water emulsion (PCE-10) has been developed based on an

organic paraffin material called RT10. In order to achieve good level of stability in the

emulsion, the HLB method was used to obtain an optimum surfactant blend consisting of two

selected surfactants (Tween 60 and Brij 52). Analysis of the thermophysical properties

revealed the particle size of the PCE-10 sample to be $3 \mu \mathrm{m}$ with a storage heat capacity of

almost twice as much as that of water and with a negligible level of sub-cooling. The PCE-10

500 freeze-thaw cycles in a pumping system without any significant sign of degradation to

particle size and heat storage capacity.

However the viscosity of the PCE-10 was found to be much higher than water which could

contribute to high pressure drop in a pumping system. Its thermal conductivity was also

found to be about $30 \%$ lower than the value for water and could influence heat transfer.

Further enhancements of these thermophysical properties are therefore recommended.

\section{Reference}

[1] Q. Zhen and H. Qian, "Overview of fan coil air conditioning system," Science Information, vol.

[2] W. Zhong, "Building air conditioning energy consumption and energy saving measures in China," MSc Master thiese, Environment engineering, MSc Chongqing University unpublished, 2004.

[3] C. Pollerberg and C. Dotsch, "Phase changing slurries in cooling and cold supply networks," in 10th International Symposium on District Heating and Cooling, 2006.

[4] Y. Xu, "Study the saving energy method for fan-coil unit system," MSc Master thiese, Construction and Civil Engineering, Xi,an University of Architecture and Technology, unpublished, 2005.

[5] L. Huang, E. Günther, C. Doetsch, and H. Mehling, "Subcooling in PCM emulsions-Part 1: Experimental," Thermochimica Acta, vol. 509, pp. 93-99, 2010.

[6] L. Royon and G. Guiffant, "Heat transfer in paraffin oil/water emulsion involving supercooling phenomenon," Energy Conversion and Management, vol. 42, pp. 2155-2161, 2001.

[7] H. Xu, R. Yang, Y. Zhang, Z. Huang, J. Lin, and X. Wang, "Thermal physical properties and key influence factors of phase change emulsion," Chinese Science Bulletin, vol. 50, pp. 188193, 2005.

[8] P. Schalbart, M. Kawaji, and K. Fumoto, "Formation of tetradecane nanoemulsion by lowenergy emulsification methods," International Journal of Refrigeration, vol. 33, pp. 1612-1624, 2010.

[9] L. Huang, M. Petermann, and C. Doetsch, "Evaluation of paraffin/water emulsion as a phase change slurry for cooling applications," Energy, vol. 34, pp. 1145-1155, 2009. 
[10] J. Vilasau, C. Solans, M. J. Gómez, J. Dabrio, R. Mújika-Garai, and J. Esquena, "Stability of oil-in-water paraffin emulsions prepared in a mixed ionic/nonionic surfactant system," Colloids and Surfaces A: Physicochemical and Engineering Aspects, vol. 389, pp. 222-229, 2011.

[11] Rubitherm Technologies GmbH. see: http://www.rubitherm.com.

[12] L. Huang, P. Noeres, M. Petermann, and C. Doetsch, "Experimental study on heat capacity of paraffin/water phase change emulsion," Energy Conversion and Management, vol. 51, pp. 1264-1269, 2010.

[13] L. Huang, "Paraffin Water Phase Change Emulsion for Cold Storage and Distribution Applications," PhD, Fraunhofer Institute UMSICHT, Ruhr-University Bochum, Electronic publication, 2009.

[15] I. Americas, The HLB system a time-saving guide to enulsifier selection. Wilmington, Delaware, US: chemmunique, 1987.

[16] K. Zhang, R. Li, Y. Lu, and P. Shang, "HLB and Surfactant selection," Journal of Shijiazhuang Vocational Technology Institute, vol. 16, pp. 20-22, 2004.

[17] K. Golemanov, S. Tcholakova, N. D. Denkov, and T. CGurkov, "Selection of Surfactants for Stable Paraffin-in-Water Dispersions, undergoing Soild-Liquid Transition of the Dispersed Particles," Langmuir, vol. 22, pp. 3560-3569, 2006.

[18] B. Wang, Y. Zhang, and D. Sun, "Preparation and application of wax emulsion," Shandong Chemical Industry, vol. 33, pp. 14-17, 2004.

[19] J. Wang and T. Zhang, "The anomalous rheological properties of wax miniemusion with mixed emulsifier," Journal of Shandong institute of light industry, vol. 11, pp. 12-14, 1997.

[20] J. Wang, "Preparation and properties of paraffin miniemulsion," Journal of Shandong institute of light industry, vol. 11, pp. 24-27, 1997.

[21] L. L. Schramm, "Emulsion, foams and suspensions," Weinheim: Wiley-VCH Verlag Gmbh \& Co, 2005.

[22] X. X. Zhang, Y. F. Fan, X. M. Tao, and K. L. Yick, "Crystallization and prevention of supercooling of microencapsulated n-alkanes," Journal of Colloid and Interface Science, vol. 281, pp. 299-306, Jan 152005.

[23] Y. Zhang, Y. Jiang, and Y. Jiang, "A simple method, the T-history method, of determining the heat of fusion, specific heat and thermal conductivity of phase-change materials," Measurement and Science Technology vol. 10, pp. 201-205, 1999. 